\title{
Stability of bubbles in a Hele-Shaw cell
}

\author{
S. Tanveer ${ }^{\text {a) }}$ and P. G. Saffman \\ Department of Applied Mathematics, California Institute of Technology, Pasadena, California 91125
}

(Received 2 March 1987; accepted 2 June 1987)

\begin{abstract}
The linear stability of steadily moving bubbles in a Hele-Shaw cell is investigated. It is shown analytically that without the effect of surface tension, the bubbles are linearly unstable with the stability operator having a continuous spectrum. For small bubbles that are circular, analytical calculations also show that any amount of surface tension stabilizes a bubble. Numerical calculations suggest that the branch of bubble solutions that, in the limit of large area, corresponds to the McLean-Saffman finger is stable for any nonzero surface tension. However, the decay rate of disturbances on the McLean-Saffman branch depends appreciably on the bubble size even for large bubbles. This suggests that the stability results on this branch cannot be immediately extrapolated to the McLean-Saffman fingers. For another branch of bubble solution, which in the limit of large area corresponds to the first of the Romero-VandenBroeck finger solutions, numerical evidence suggests that it is unstable to one symmetric and one antisymmetric mode for any surface tension. The symmetric unstable mode tends to break the tip of the bubble and the growth rate of this mode is unaffected by further increase in bubble size, once the bubble is large enough. This suggests that there is an analogous instability for the finger, and this agrees with the numerical findings of Kessler and Levine [Phys. Rev. A 33, 2632 (1986) ]. Agreement is noted in the quantitative comparison of the growth rate with the predictions [Tanveer, Phys. Fluids 30, 2318 (1987)] on the limiting growth rate for the symmetric unstable mode for the first Romero-Vanden-Broeck branch of finger solution.
\end{abstract}

\section{INTRODUCTION}

Two phase flow in a Hele-Shaw cell has attracted a lot of attention in recent years in view of its relation to a multitude of problems in oil reservoir engineering, 'dendritic crystal growth, ${ }^{2}$ and to problems of pattern selection, phase transition, and formation of fractal structures. ${ }^{3}$ A large part of the research has been devoted to the study of a single steadily moving finger ${ }^{4-12}$ since the pioneering work of Saffman and Taylor. ${ }^{13}$ The linear stability of the finger has been the subject of some controversial claims. ${ }^{4.7}$ In another paper, ${ }^{12}$ an analytical theory is presented to investigate the limiting growth or decay rate for different modes for the SaffmanTaylor finger as surface tension tends to zero and it is shown that the spectrum of the stability operator for zero surface tension and the limit of zero surface tension are different. The numerically computed values of the limiting eigenvalues based on this analytic theory confirms the conjecture of Kessler and Levine ${ }^{7}$ that the McLean-Saffman branch of finger solutions remains stable for any nonzero surface tension, while other branches calculated by Romero ${ }^{5}$ and Vanden-Broeck ${ }^{6}$ are unstable. Kessler and Levine ${ }^{7}$ calculate the eigenvalues of the stability operator for nonzero surface tension. When surface tension tends to zero, more and more terms are necessary in the truncation of the basis representation for the unknown functions and this makes it difficult to obtain reliable results. However, extrapolating from the trend, they conjecture that the McLean-Saffman branch of finger solution remains stable for any nonzero surface tension.

\footnotetext{
a) Permanent address: Math Department, Virginia Polytechnic Institute and State University, Blacksburg, Virginia 24060.
}

In this paper, we investigate the analogous problem of the linear stability of a steadily moving bubble in a HeleShaw cell. These steady states were computed before by Tanveer $^{14,15}$ for nonzero surface tension. Earlier, Taylor and Saffman ${ }^{16}$ found exact solutions for zero surface tension for bubbles symmetric about the channel centerline. Tanveer ${ }^{15}$ and Kadanoff ${ }^{17}$ find nonsymmetric bubble solutions as well. For given bubble area, channel width, and flow conditions at infinity, the bubble velocity and the ratio of the viscous fluid passing over to that passing under the bubble in the frame of the moving bubble remain arbitrary. However, as soon as surface tension is introduced, only symmetric bubbles with a discrete set of bubble velocities were found to be possible in general for any given surface tension. ${ }^{14,15}$ The existence of nonsymmetric bubbles is not completely ruled out even though none could be found numerically; however, it is shown that they cannot exist as a continuation from the symmetric family of bubble solutions. The effect of surface tension is similar here to that on the nonsymmetric continuum of finger solutions of arbitrary width where previous work $^{8-10,18}$ shows that only fingers with specific widths and symmetry are possible for nonzero surface tension. Different branches of steady state bubbles were found. ${ }^{14,15}$ The bubbles corresponding to one solution branch, called the main branch, ${ }^{14}$ reduce to circular shapes in the limit of small area, and, in the limit of large area, the front half of the bubble tends to the finger solutions of McLean and Saffman. ${ }^{4}$ Another branch calculated by Tanveer ${ }^{15}$ reduced to the first of the Romero-Vanden-Broeck branch of fingers ${ }^{5,6}$ in the limit of large area (the branches being numbered in order of increasing finger widths). Subsequently, we found numerically one more branch that corresponds to the second RomeroVanden-Broeck solution branch and it is likely that there are 
others, perhaps, a discrete infinity of such solutions similar to the finger.

In this paper, we first consider the stability features of a small steady bubble analytically. For zero surface tension, the spectrum is the entire complex plane both for the symmetric and antisymmetric disturbances implying that the bubble is unstable. However, when any amount of surface tension is introduced, the bubble stabilizes and the stability operator has a discrete spectrum with each eigenvalue varying linearly with surface tension. Except for the trivial translation mode, none of the eigenmodes in the zero surface tension analysis are recovered in the limit of zero surface tension. A formal perturbation series in powers of surface tension is constructed for each of these modes and is found to be consistent without any constraint on the spectrum. We conclude that zero surface tension modes, except for the trivial translation mode, are perturbed by surface tension such that transcendentally small terms in surface tension arise that distort the bubble boundary into a nonanalytic curve.

Second, we consider analytically the zero surface tension spectrum for symmetric disturbances of a finite bubble, not necessarily small, and find it to be the entire complex plane once again. For small surface tension, a consistent regular perturbation series in powers of surface tension appears to be possible without any restrictions on the spectrum, in apparent contradiction to numerical evidence for small nonzero surface tension. It appears to us that the transcendentally small terms in surface tension are again responsible for the selection of a discrete set of (in general stable) eigenmodes from the continuum of zero surface tension modes. This conjecture is based on our previous stability analysis ${ }^{12}$ of the finger.

Third, we investigate the spectrum of the stability operator for symmetric and antisymmetric disturbances over a range in surface tension for bubbles of different areas on different branches of steady solutions. It is found that for values of surface tension greater than some small number, below which numerically the eigenvalues become too unreliable, the bubbles on the McLean-Saffman branch are stable to both symmetric and nonsymmetric disturbances. Extrapolation for smaller values of the surface tension suggests that any finite size bubble on the McLean-Saffman branch remain stable in the limit of zero surface tension. For the first Romero-Vanden-Broeck branch, there is one unstable symmetric mode and one unstable antisymmetric mode. From limited numerics on the second Romero-Vanden-Broeck branch, it appears that there are two such unstable modes for each of the symmetric and antisymmetric disturbances. This is consistent with the numerical findings of Kessler and Levine. ${ }^{19}$

Extrapolating the unstable symmetric eigenvalue for large bubbles on the first Romero-Vanden-Broeck branch to zero surface tension, we get a limiting value that is in agreement with the prediction from the analytical theory ${ }^{12}$ for the first Romero-Vanden-Broeck branch of finger solution. A power law dependence of this eigenvalue on surface tension is also noted to be in correspondence with the previous theory. ${ }^{12}$ For bubbles on the McLean-Saffman branch, the symmetric eigenmodes, at least the first few least stable ones, affect only the rear of the bubble and there are no analogous disturbances on the finger since in the stability analysis of the finger, all the disturbances are usually assumed to decay at the far end. It is also found that on this branch, the least stable eigenvalues depend appreciably on the bubble size, as expected. The stability properties of the finger on the McLean-Saffman branch cannot therefore be immediately deduced from the study of long bubbles. However, from the study of the zero surface tension limit ${ }^{12}$ and earlier investigations ${ }^{7}$ for small surface tension, the McLean-Saffman finger appears to be linearly stable for any nonzero surface tension. The results here for the McLean-Saffman branch of bubble solutions do not contradict this finding.

\section{STABILITY OF SMALL BUBBLES}

In the small bubble area limit, the effect of the walls can be ignored and the bubble corresponding to the main branch of solution ${ }^{14}$ is circular and moves with velocity $U=2 \mathrm{~V}$, where $V$ is the velocity of the more viscous fluid at infinity. We will consider the stability of this steady bubble solution to infinitesimal disturbances that preserve the flux of fluid $V$ at infinity. Without any loss of generality, $V$ will be chosen to be unity. Further, in this section for algebraic convenience, we will take the steady bubble radius $R_{b}$ to be unity, in contrast to the choice in other sections where the cell half-width $a$ is set to unity. This is equivalent to nondimensionalizing all the variables of interest using appropriate combinations of $V$ and the bubble radius. The scaling in this section implies that $a \rightarrow \infty$, i.e., the cell walls recede to infinity.

We switch to a frame that moves with the steady bubble. In the steady state flow, the viscous fluid streams past the circular bubble from right to left with unit velocity at $\infty$. The equations of motion governing unsteady motion are the dynamic boundary condition

$$
\phi+2 x=\left(b^{2} T / 12 \mu V R_{b}^{2}\right) \kappa
$$

and the kinematic boundary condition

$$
\frac{D}{D t}[r-1-\epsilon F(\theta, t)]=0,
$$

each satisfied on the boundary of the unsteady bubble

$$
r=1+\epsilon F(\theta, t),
$$

where $r$ is the radial distance from the center of the bubble, $\theta$ is the angle with respect to the positive $x$ axis, and $\phi$ is the velocity potential that satisfies Laplace's equation outside the bubble. Here, $b$ is the cell gap, $T$ is the surface tension, $\mu$ denotes the viscosity of the viscous fluid, and $\epsilon$ is an infinitesimal parameter. The boundary condition on $\phi$ at $\infty$ is

$$
\phi \sim-x+O(1 / r),
$$

where we assume that there are no logarithmic terms (sources). We decompose the velocity potential

$$
\phi=\phi_{0}(x, y)+\epsilon \phi_{1}(x, y, t)
$$

where $\phi_{0}$ is the potential corresponding to the steady flow and is

$$
\phi_{0}=-(r+1 / r) \cos \theta .
$$

Linearizing the equations of motion for small $\epsilon$ and assuming the finger boundary to be analytic, we can shift the 
boundary conditions back to the original circular boundary by standard expansion. Assuming a $e^{\sigma t}$ time dependence for the dependent variables, one finds that the equations determining linear stability are given by the following boundary conditions on $r=e^{i \theta}$ :

$$
\begin{aligned}
& \phi_{1 r}-2 \cos \theta F=\sigma F+2 \sin \theta F_{\theta}, \\
& 2 \cos \theta F+\phi_{1}=-\mathscr{C}\left(F+F_{\theta \theta}\right),
\end{aligned}
$$

where a subscript $\theta$ denotes differentiation with respect to $\theta$ and $\mathscr{C}$ is a surface tension parameter defined by

$$
\mathscr{C}=b^{2} T / 12 \mu V R_{b}^{2} \text {. }
$$

It is not hard to see from the boundary conditions (7) and (8) that any eigenfunctions comprising the pair $\left(\phi_{1}, F\right)$ are either even or odd functions of the angular variable $\theta$, corresponding to symmetric or antisymmetric disturbances. In fact, this result carries over to arbitrary size bubbles that are symmetric about the channel centerline.

For symmetric disturbances, we write

$$
\begin{aligned}
& F=\sum_{0}^{\infty} a_{n} \cos n \theta, \\
& \phi_{1}(r, \theta)=\sum_{0}^{\infty} b_{n} r^{-n} \cos n \theta .
\end{aligned}
$$

Substituting into (7) and (8), we find the following recurrence relation:

$$
\begin{aligned}
& \sigma a_{0}=0, \\
& a_{2}=\sigma a_{1} / 2,
\end{aligned}
$$

and for $n \geqslant 2$,

$$
a_{n+1}=(1 / 2 n)\left[\sigma+\mathscr{C} n\left(n^{2}-1\right)\right] a_{n} .
$$

For zero surface tension, we find that $\sigma$ can be any value, real or complex, and for any nonzero $\sigma$, the corresponding symmetric eigenfunction is given by

$$
F=\sum_{0}^{\infty} \frac{\sigma^{n}}{2^{n} n !} \cos (n+1) \theta .
$$

When $\sigma=0$, we can only have $a_{0}$ and $a_{1}$ nonzero. When $a_{1}$ is the only nonzero element, the eigenmode corresponds to a pure translation along the channel, call this the trivial mode. When $a_{0}$ is the only nonzero element, we have a uniform radial expansion of the bubble. This is an eigenmode corresponding to zero eigenvalue because there is a neighboring steady solution corresponding to a circular bubble of radius $1+\epsilon$ that moves with the same velocity as the bubble of radius 1 because the wall effects are ignored. From (15), it is clear that for $\mathscr{C}=0$, the bubble is unstable with a continuous spectrum for the stability operator. We will see in the next section that this is true for bubbles of any size when surface tension is totally neglected.

Now, if $\mathscr{C}$ is nonzero, it is clear from the recurrence relation (14) that in general $a_{n}$ grows with $n$ for large $n$. This is unacceptable since the infinite series representation in (10) will not be convergent. Thus the series in (10) must truncate, implying that the nonzero eigenvalues form a discrete set of real negative values given by $\left\{\sigma_{m}\right\}$, where

$$
\sigma_{m}=-\mathscr{C} m\left(m^{2}-1\right),
$$

with the integer $m$ ranging from 2 to $\infty$. Thus the bubble is stable when any amount of surface tension is introduced. The eigenfunctions corresponding to each of these nonzero eigenvalues are readily determined from the recurrence relation [ $(13)$ and (14)]. Corresponding to the smallest nonzero eigenvalue $\sigma_{2}$, the eigenfunction is

$$
F=\cos \theta+\frac{1}{2} \sigma_{2} \cos 2 \theta \text {. }
$$

Now, we consider antisymmetric disturbances. Then

$$
\begin{aligned}
& F=\sum_{1}^{\infty} a_{n} \sin n \theta, \\
& \phi_{1}(r, \theta)=\sum_{1}^{\infty} b_{n} r^{-n} \sin n \theta .
\end{aligned}
$$

Substituting back into (7) and (8), we obtain the same recurrence relation (14) for $a_{n}$, where it is now valid for $n \geqslant 1$. For zero surface tension, once again, we find no restriction on the eigenvalue $\sigma$. When surface tension is nonzero, once again (16) determines the discrete set of eigenvalues, where $m$ in this case ranges from 1 to $\infty$. For $m=1, \sigma_{m}=0$ and the corresponding eigenvector is $F=\sin \theta$. This actually corresponds to a translation of the bubble in the direction perpendicular to the channel flow. This is a neutral eigenvector in this case because we have ignored the effect of the cell walls on the bubble. When the wall effect is included, there is no such neutral eigenvector as numerical results for finite bubbles will show. This is in accordance with Tanveer's ${ }^{15}$ steady state calculations, where it is shown that there are no nonsymmetric steady bubble solutions that exist as a continuation of the family of symmetric bubbles. For $m=2$, the eigenfunction is given by

$$
F=\sin \theta-\frac{1}{2} \sigma_{2} \sin 2 \theta .
$$

Other eigenfunctions can be similarly obtained using the recursion relations. Thus we find that, as in the case of symmetric disturbances, any amount of surface tension stabilizes the bubble.

It is interesting to note that as the surface tension decreases, the zero surface tension eigenmodes given by (15) satisfy the nonzero surface tension stability equations (7) and (8) with arbitrarily small error; yet there is for general $\sigma$ no exact eigenmode for nonzero surface tension close to (15). This is exactly the same situation that occurs in the stability analysis of fingers. ${ }^{12}$ As happens in that case, perturbations of the zero surface tension bubble modes (15) to include effects of surface tension lead to shapes that are nonanalytic, and are therefore excluded by the nonzero surface tension analysis that implicitly assumes that the bubble boundary is smooth. This nonanalytic behavior is transcendentally small in the surface tension and therefore not seen in a regular perturbation series analysis as is now described.

We expand an eigenmode $F$ in a perturbation series in $\mathscr{C}$,

$$
F=\sum_{0}^{\infty} \mathscr{C}^{n} F_{n}
$$

and use a similar series for $\phi_{1}$. Consider only the symmetric modes. The leading term $F_{0}$ is given by (15). To find $F_{1}$, if we expand it in a Fourier cosine series (10), and find that the coefficients $a_{n}$ in this case satisfy the recurrence relation 


$$
a_{n+1}=\frac{\sigma}{2 n} a_{n}+\frac{(n+1) \sigma^{n-1}}{2^{n}(n-1) !},
$$

for $n \geqslant 2$, the resulting series for $F_{1}$ can be shown to converge for any $\sigma$ and describes a smooth function. Similarly, higherorder perturbation terms in (21) can be found that are smooth for any value of $\sigma$. Thus the perturbation in surface tension analysis does not impose any restrictions on $\sigma$, and seems to suggest that the bubble is unstable in contradiction to our exact results (16). The only way to resolve this contradiction is to realize that the expansion (21) is asymptotic and not convergent, and that transcendentally small terms in the surface tension, which are not resolved by the expansion (21), will not allow the perturbation to the boundary to be smooth unless $\sigma$ is restricted to one of the values given by (16). A calculation to demonstrate this explicitly is left for future work.

\section{STABILITY OF ARBITRARY SIZED BUBBLES FOR ZERO SURFACE TENSION}

In this section, we will be limited to symmetric disturbances about the channel centerline. We now take the cell half-width $a$ and the velocity $V$ at $\infty$, taken to be independent of time, to be unity so that all our variables are nondimensionalized using appropriate combinations of $a$ and $V$. We move to the frame that moves to the right with velocity $U$, the velocity of the steady bubble. The flow geometry in this frame in the $z$ plane is shown in Fig. 1. Only the flow in the upper half $z$ plane will concern us in this section because of the assumed symmetry of the disturbance. The dynamic boundary condition on the bubble for zero surface tension is

$$
\phi+U x=\text { const, }
$$

where the constant appearing in (23) can be a function of time. On the cell wall, $y=1$, the streamfunction satisfies the condition

$$
\psi=-(U-1),
$$

and on the line of symmetry $y=0$, outside the bubble, we have

$$
\psi=0
$$

Except for some minor changes in constant multiplicative factors made for algebraic convenience, the stability formulation in this section closely follows the steady state formulation of Tanveer. ${ }^{14}$ At any time $t$, consider the conformal map
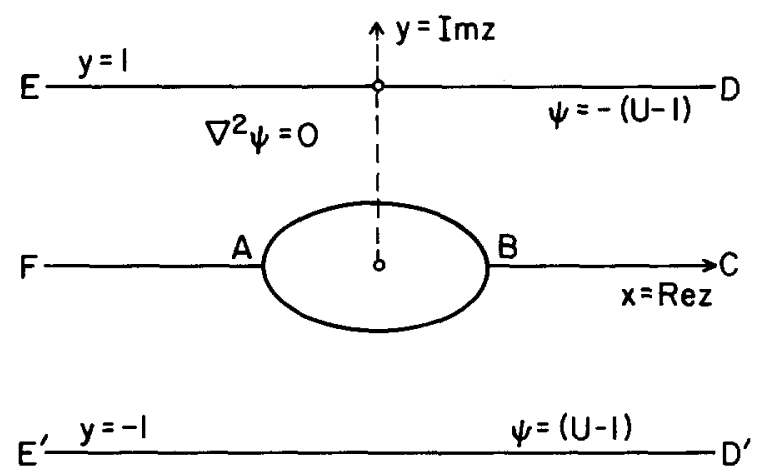

FIG. 1. The physical flow domain (the $z$ plane).

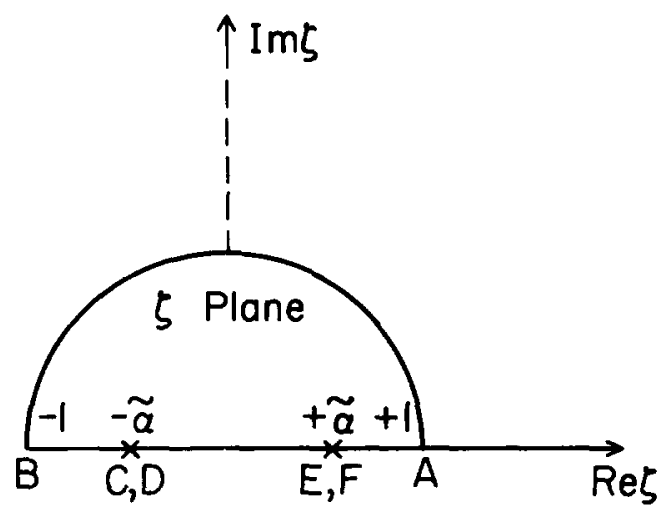

FIG. 2. The semicircular $\zeta$ plane used in analysis in Sec. III.

$z(\zeta, t)$ of the upper half-flow domain in the $z$ plane into the unit semicircle in the $\zeta$ plane (Fig. 2), so that $\zeta=-1$ and +1 correspond to the front and rear stagnation points on the bubble. The third degree of freedom of the Riemann mapping theorem is utilized by requiring that the image of $z=-\infty$ and $+\infty$ are located at the same distance from the origin at $\zeta= \pm \widetilde{\alpha}$ on the real $\zeta$ diameter. The upper half bubble boundary at any time $t$ corresponds to the arc of the unit semicircle in the $\xi$ plane. The flow domain in the $\xi$ plane is therefore fixed in time although $\widetilde{\alpha}$ is in general a function of $t$, which has to be determined as part of the problem. We decompose $\widetilde{\alpha}$ into

$$
\widetilde{\alpha}=\alpha+\widetilde{\alpha}_{1}(t),
$$

where $\alpha$ is the time-dependent value for the steady bubble. We also decompose the mapping function $z(\xi, t)$ into

$$
z=z_{0}(\zeta, \tilde{\alpha})+k_{s} \tilde{f}(\zeta, t),
$$

where

$$
z_{0}=\frac{1}{\pi} \ln \left(\frac{\zeta-\widetilde{\alpha}}{\zeta+\widetilde{\alpha}}\right)+\frac{1}{\pi}\left(\frac{2}{U}-1\right) \ln \left(\frac{1+\zeta \widetilde{\alpha}}{1-\zeta \widetilde{\alpha}}\right)
$$

and

$$
k_{s}=(2 \alpha / \pi U)\left[U\left(1+\alpha^{2}\right)-2 \alpha^{2}\right] .
$$

We note that $z_{0}$ depends on time $t$ through $\tilde{\alpha}$. The function $z_{0}(\zeta, \alpha)$ corresponds to the Saffman-Taylor exact bubble solutions as discussed earlier. ${ }^{14}$ In this case both the parameters $U$ and $\alpha$ are arbitrary.

For the general time-dependent flow, the function $\tilde{f}$ is analytic everywhere in and on the unit semicircle if the boundary of the finger is assumed to be smooth. Further, using the known value of $\operatorname{Im} z$ on the upper cell wall and the line of symmetry, we find that for real $\zeta$ in the interval $[-1,1]$,

$$
\operatorname{Im} \tilde{f}=0 \text {. }
$$

For zero surface tension, the steady state corresponds to $\tilde{f}=0$ as discussed earlier. ${ }^{14}$

We decompose the complex velocity potential $W=\phi+i \psi$ into

$$
W=W_{0}(\zeta, \widetilde{\alpha})+U k_{s} \widetilde{W}(\zeta, t),
$$

where 


$$
W_{0}=-\frac{(U-1)}{\pi} \ln \left(\frac{(\zeta-\widetilde{\alpha})(1-\widetilde{\alpha} \zeta)}{(\zeta+\widetilde{\alpha})(1+\widetilde{\alpha} \zeta)}\right) .
$$

We note that $W_{0}$ also depends on $t$ through $\widetilde{\alpha}$. Here $W_{0}(\zeta, \alpha)$ is the complex velocity potential for the steady problem, and $\widetilde{W}$ is an analytic function of $\zeta$ inside the unit circle with

$$
\operatorname{Im} \widetilde{W}=0
$$

on the real $\zeta$ diameter because of (24) and (25). It is also analytic on the circular arc of the semicircle if we assume that the boundary is smooth. For the steady problem, it is identically zero.

Using the fact that $\operatorname{Re}\left(W_{0}+U z_{0}\right)=0$ on the unit semicircular arc the dynamic boundary condition (23) is equivalent to the following condition on the arc of the semicircle $\xi=e^{i v}$ :

$$
\operatorname{Re}(\tilde{f}+\widetilde{W})=0 .
$$

From (30), (33), and (34), it follows that

$$
\tilde{f}+\widetilde{W}=0
$$

everywhere.

Now we consider the kinematic condition. Since the bubble boundary in this formulation corresponds to the fixed circular arc of the unit semicircle at all times, we have

$$
\frac{D}{D t} \ln \hat{\rho}(x, y, t)=0,
$$

on $|\zeta|=1$, where $\hat{\rho} e^{i v}=\zeta$ in the polar representation and $D / D t$ represents the time derivative in the frame of a moving particle $(x(t), y(t))$ on the bubble surface in the physical plane. Note that in (36), the triple $(x, y, t)$ is independent, while $(\ln \hat{\rho}, v, t)$ is dependent. This is a one to one transformation. On inverting the transformation, we find that (36) is equivalent to

$$
\operatorname{Re}\left(\zeta W^{\prime}-\zeta^{*} z^{\prime *} z_{t}\right)=0
$$

on the upper half unit semicircular arc in the $\zeta$ plane, where the prime denotes the derivative with respect to $\zeta$ keeping $t$ fixed, a subscript $t$ denotes the derivative with respect to time, keeping $\zeta$ fixed, and a superscript $*$ denotes complex conjugation. Note that (37) does not depend on the nature of the work plane $\xi$ except that the arc of a circle should correspond to the free boundary at all times. Equation (37) will also be used in the next section, where the physical flow region in the $\zeta$ plane corresponds to the annular region between two concentric circles.

Equations (35) and (37) determine $z$ and $W$ as functions of $\zeta$ and $t$ for the general initial value problem when the initial conditions have symmetry about the channel centerline. For the linearized stability problem we make the usual assumptions of small disturbances about the steady state by assuming

$$
\begin{aligned}
& \tilde{f}=\epsilon F(\zeta, t), \\
& \widetilde{W}=\epsilon \omega(\zeta, t), \\
& \widetilde{\alpha}=\alpha+\epsilon \alpha_{1}(t),
\end{aligned}
$$

where $\epsilon$ is infinitesimal. Using (27), (28), (31), and (32), and inserting relations (35) and (38)-(40) into (37) we find that the order $\epsilon$ equation can be put into the following form on $\zeta=e^{i v}$ :
$\operatorname{Re}\left(\zeta F^{\prime}+\frac{\zeta\left(\zeta^{2}-p^{2}\right)}{\left(1-\alpha^{2} \zeta^{2}\right)\left(\zeta^{2}-\alpha^{2}\right)} F_{\tau}+R(\zeta) \alpha_{1 \tau}\right)=0$,

where

$$
p^{2}=\left[U\left(1+\alpha^{2}\right)-2\right] /\left[U\left(1+\alpha^{2}\right)-2 \alpha^{2}\right]
$$

and

$$
\tau=U t / k_{s}
$$

is the scaled time. We note that $p^{2}$ is in the interval $(0,1)$ when $U\left(1+\alpha^{2}\right)>2$ and we will assume this to be the case since it is found to be true for all the steady state solutions calculated earlier. ${ }^{14,15}$ We define $R(\zeta)$ to be an analytic function of $\zeta$ by the following boundary conditions:

$$
\operatorname{Im} R=0
$$

on the real diameter and on the semicircular $\operatorname{arc}, \zeta=e^{i v}$,

$$
\begin{aligned}
\operatorname{Re} R= & \frac{2}{\pi k_{s}} \operatorname{Re}\left[\left(\frac{\zeta\left(\zeta^{2}-p^{2}\right)}{\left(1-\alpha^{2} \zeta^{2}\right)\left(\zeta^{2}-\alpha^{2}\right)}\right)\right. \\
& \left.\times\left(-\frac{\zeta}{\zeta^{2}-\alpha^{2}}+\frac{\zeta\left(\alpha^{2}-p^{2}\right)}{\left(1-\alpha^{2} \zeta^{2}\right)\left(1-\alpha^{2} p^{2}\right)}\right)\right] .
\end{aligned}
$$

We note that the function within the parentheses in (41) has simple pole singularities at $\xi= \pm \alpha$. Subtracting these singularity terms and adding back regular terms with the same real part on the arc of the unit circle, we find that (41) can be reduced to

$$
\begin{aligned}
\zeta F^{\prime} & +\frac{\zeta\left(\zeta^{2}-p^{2}\right)}{\left(1-\alpha^{2} \zeta^{2}\right)\left(\zeta^{2}-\alpha^{2}\right)} F_{\tau}+R \alpha_{1 \tau} \\
& +\frac{\left(\alpha^{2}-p^{2}\right)\left(\zeta^{2}-1\right)}{2\left(1-\alpha^{4}\right)} \\
& \times\left(\frac{F_{\tau}(\alpha)}{(\zeta-\alpha)(1-\alpha \zeta)}+\frac{F_{\tau}(-\alpha)}{(\zeta+\alpha)(1+\alpha \zeta)}\right)=0 .
\end{aligned}
$$

The parameter $\alpha_{1}$ is determined in terms of $F$ by evaluating (44) at $\xi=0$. This gives us

$$
\frac{\left(\alpha^{2}-p^{2}\right)}{2 \alpha\left(1-\alpha^{4}\right)}\left[F_{\tau}(\alpha)-F_{\tau}(-\alpha)\right]+R(0) \alpha_{1 \tau}=0 .
$$

Eliminating $\alpha_{1}$, (44) reduces to

$$
\begin{aligned}
F^{\prime}+ & \frac{\left(\zeta^{2}-p^{2}\right)}{\left(1-\alpha^{2} \zeta^{2}\right)\left(\zeta^{2}-\alpha^{2}\right)} F_{\tau} \\
& =F_{\tau}(\alpha) \frac{P_{1}}{\zeta-\alpha}+F_{\tau}(-\alpha) \frac{P_{2}}{\zeta+\alpha},
\end{aligned}
$$

where

$$
P_{1}=\frac{\left(\alpha^{2}-p^{2}\right)}{2\left(1-\alpha^{4}\right)}\left(-\frac{\left(\zeta^{2}-1\right)}{\zeta(1-\alpha \zeta)}+\frac{(\zeta-\alpha) R(\zeta)}{\alpha R(0) \zeta}\right)
$$

and

$$
P_{2}=\frac{\left(\alpha^{2}-p^{2}\right)}{2\left(1-\alpha^{4}\right)}\left(-\frac{\left(\zeta^{2}-1\right)}{\zeta(1+\alpha \zeta)}-\frac{(\zeta+\alpha) R(\zeta)}{\alpha R(0) \zeta}\right) .
$$

We note that each of $P_{1}$ and $P_{2}$ are analytic functions within the unit circle. The initial value problem in (46) can be explicitly solved for the special case of $\alpha=p$, in which case the right-hand side of (46) is zero. We find that

$$
F(\zeta, t)=F_{0}\left(\frac{(1+\alpha \zeta) e^{-2 \alpha t}-(1-\alpha \zeta)}{\alpha(1+\alpha \zeta) e^{-2 \alpha t}+\alpha(1-\alpha \zeta)}\right),
$$


where $F_{0}(\zeta)=F(\zeta, 0)$ is the initial value. It is clear from (49) that there are initial conditions for which $F$ grows without bound. This is because the argument of $F_{0}$ in (49) can exceed 1 in absolute value and $F_{0}$ can have singularities outside the unit circle. Thus the Saffman-Taylor bubble is linearly unstable for $U=2$ when surface tension is completely neglected. In the general case of arbitrary $U$, i.e., for $\alpha \neq p$, the initial value problem can only be solved implicitly which is not very convenient for analysis. If instead, we consider the eigenvalue problem where each of the functions are assumed to have a $e^{\sigma t}$ dependence, then (46) reduces to a firstorder ordinary differential equation (ODE) involving $\sigma$, which is readily solved using integrating factors. We will carry on with the same notation $F$ for the eigenfunction as for the original function to avoid proliferation of symbols. The context is clear and hopefully will not cause any confusion. When $\operatorname{Re} \sigma \geqslant 0$, the eigenvector $F(\zeta)$ is

$$
\begin{aligned}
F= & \sigma H^{-1}(\zeta) F(\alpha) \int_{\alpha}^{\zeta} d \zeta^{\prime} P_{1}\left(\zeta^{\prime}\right) \frac{H\left(\zeta^{\prime}\right)}{\left(\zeta^{\prime}-\alpha\right)} \\
& +\sigma H^{-1}(\zeta) F(-\alpha) \\
& \times \int_{\alpha}^{\zeta} d \zeta^{\prime} P_{2}\left(\zeta^{\prime}\right) \frac{H\left(\zeta^{\prime}\right)}{\left(\zeta^{\prime}+\alpha\right)}
\end{aligned}
$$

where

$$
H(\zeta)=\left(\frac{1+\alpha \zeta}{1-\alpha \zeta}\right)^{\sigma d_{1}}\left(\frac{\alpha-\zeta}{\zeta+\alpha}\right)^{\sigma d_{2}}
$$

where

$$
d_{1}=\left(1-p^{2} \alpha^{2}\right) / 2 \alpha\left(1-\alpha^{4}\right)
$$

and

$$
d_{2}=\left(\alpha^{2}-p^{2}\right) / 2 \alpha\left(1-\alpha^{4}\right) .
$$

In the case when $\operatorname{Re} \sigma<0$, the eigenfunction is

$$
\begin{aligned}
F= & \sigma H^{-1}(\zeta) F(\alpha) \int_{-\alpha}^{\zeta} d \zeta^{\prime} P_{1}\left(\zeta^{\prime}\right) \frac{H\left(\zeta^{\prime}\right)}{\left(\zeta^{\prime}-\alpha\right)} \\
& +\sigma H^{-1}(\zeta) F(-\alpha) \int_{-\alpha}^{\zeta} d \zeta^{\prime} P_{2}\left(\zeta^{\prime}\right) \frac{H\left(\zeta^{\prime}\right)}{\left(\zeta^{\prime}+\alpha\right)}
\end{aligned}
$$

At first sight it may appear that the expression in (50) has singularities at $\zeta= \pm \alpha$, which are not allowable. However, on closer inspection, we find that the singularities inside and outside the integrals cancel each other out precisely at $\xi=\alpha$. At $\zeta=-\alpha$, the integrand as it appears in (50) is not integrable. However, we follow the standard trick of subtracting out the singularity from the integrand in a form that is analytically integrable. It is possible to choose $F(-\alpha)$ in terms of $F(\alpha)$ in such a way that $F$ is regular at $\zeta=-\alpha$ as well. When $0<d_{2} \operatorname{Re} \sigma<1$, this condition between $F(-\alpha)$ and $F(\alpha)$ is given by

$$
\begin{aligned}
\sigma F( & -\alpha) \int_{\alpha}^{-\alpha} d \zeta^{\prime}\left[P_{2}\left(\zeta^{\prime}\right) \frac{H\left(\zeta^{\prime}\right)}{\left(\zeta^{\prime}+\alpha\right)}\right. \\
& \left.-\frac{P_{2}(-\alpha)(2 \alpha)^{\sigma d_{2}}}{\left(\zeta^{\prime}+\alpha\right)^{1+\sigma d_{2}}}\left(\frac{1-\alpha^{2}}{1+\alpha^{2}}\right)^{\sigma d_{1}}\right] \\
& +\frac{P_{2}(-\alpha) F(-\alpha)}{d_{2}}\left(\frac{1-\alpha^{2}}{1+\alpha^{2}}\right)^{\sigma d_{1}} \\
& +\sigma F(\alpha) \int_{-\alpha}^{\alpha} d \zeta^{\prime} P_{1}\left(\zeta^{\prime}\right) \frac{H\left(\zeta^{\prime}\right)}{\left(\zeta^{\prime}-\alpha\right)}=0 .
\end{aligned}
$$

We can find similar expressions when $d_{2} \operatorname{Re} \sigma \geqslant 1$; however, we do not write them down since, for any finite $\sigma$, the condition $d_{2} \operatorname{Re} \sigma<1$ will be satisfied for small enough surface tension, since $d_{2}$ tends to zero as $p \rightarrow \alpha$. Similar relations between $F(\alpha)$ and $F(-\alpha)$ exist that ensure that $F(\xi)$ given by (54) for $\operatorname{Re} \sigma<0$ is regular at $\xi=\alpha$. The important point here is that there is no restriction on $\sigma$ except that it cannot be purely imaginary since in that case we cannot find any solutions to (46) analytic within the unit circle except in the special case of $U=2$. We conclude that in general as well as for the special case of $U=2$, the bubble is unstable when surface tension is totally ignored, with the stability operator having a continuous spectrum. As $U$ tends to 2, i.e., $\alpha \rightarrow p$, it is not difficult to show that each of (50) and (54) reduces to

$$
F=[(1-\alpha \zeta) /(1+\alpha \zeta)]^{\sigma /(2 \alpha)}
$$

where constant multiplicative factors have been ignored.

Now, for nonzero surface tension, it is possible to expand the eigenfunction $F$ in a perturbation series containing powers of surface tension by treating $p$ as if it were independent of surface tension. At each stage of perturbation, we have a first-order ODE as in (46) with a different right-hand side. Multiplying the equation by the integrating factor $H$, we can once again write the first-order perturbation solution in terms of an integral and the function is seen to be regular inside the unit circle without any constraint on $\sigma$. We do not present the details of this perturbation here because it is similar, though a little more complicated, to the expression for the first-order perturbation of modes for the finger presented in Tanveer. ${ }^{12}$ The conclusions from a regular perturbation analysis might lead one to believe that the bubble is unstable for small surface tension. However, numerical results seem to suggest that the spectrum is discrete and stable. Thus in this case, as in the case of small bubbles, it appears that the effect of transcendentally small terms in surface tension is important. We believe the transcendentally small terms fail to be smooth on the bubble boundary unless $\sigma$ is restricted to some specific values. The analytic determination of these terms can be done using the previous method ${ }^{12}$ for the finger. However, this involves a lot of algebra and is left for the future.

\section{FORMULATION FOR NUMERICAL DETERMINATION OF STABILITY}

For the general case of arbitrary bubble size and surface tension, it does not appear possible to compute the eigenfunction and eigenvalues in closed form. We resort to numerical determination. For symmetric disturbances, it is possible to carry this out using the power series representation for each of the functions $F$ and $\omega$ introduced in the last section. A similar method was used to compute the steady state function $f$ before. ${ }^{14}$ However, this is not convenient when the bubble size is large ${ }^{15}$ because of the slow decay of the Taylor series coefficient. An alternative formulation is presented here which is an extension of the formulation ${ }^{15}$ of the steady state problem. We choose the work plane, call it the $\zeta$ plane, to be the annular region between concentric circles, shown in Fig. 3. In this case, no flow symmetry is assumed and the flow region in the physical region outside 


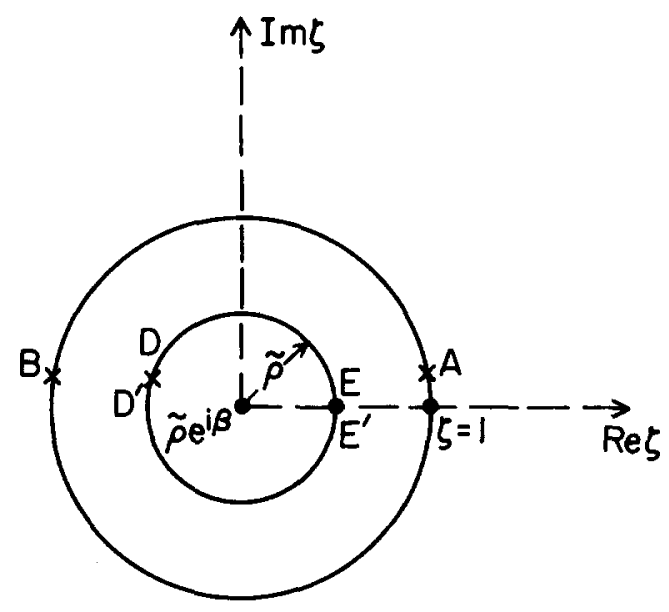

FIG. 3. The $\zeta$ plane for numerical determination in Sec. IV.

the bubble, and between the two walls, is mapped to the annular region in the $\zeta$ plane such that $z=-\infty$ is mapped to $\zeta=\hat{\rho}, z=+\infty$ to $\zeta=\hat{\rho} e^{i \bar{\beta}}$. The bubble boundary corresponds to the unit circle in the $\zeta$ plane, and the cell walls correspond to the circle of radius $\hat{\rho}$. In general, $\hat{\rho}$ and $\tilde{\beta}$ are functions of time. We decompose the mapping function $z(\zeta)$ into

$$
z(\zeta)=z_{0}(\zeta, \tilde{\rho}, \tilde{\beta})+f(\zeta, \tilde{\rho})+\tilde{z}_{1}(\zeta, t),
$$

where $z_{0}$ is now defined as

$$
\begin{aligned}
z_{0}= & \frac{-i(\pi-\tilde{\beta})}{\pi}+\frac{2}{\pi} \\
& \times \log \left(e^{-i \tilde{\beta} / 2} \frac{\theta_{1}\left((-i / 2) \ln \zeta+(i / 2) \ln \tilde{\rho}, \tilde{\rho}^{2}\right)}{\theta_{1}\left((-i / 2) \ln \zeta-\tilde{\beta} / 2+(i / 2) \ln \tilde{\rho}, \tilde{\rho}^{2}\right)}\right) \\
& +\frac{2(U-2)}{\pi U} \\
& \times \log \left(e^{i \bar{\beta} / 2} \frac{\theta_{1}\left((-i / 2) \ln \zeta-(i / 2) \ln \tilde{\rho}, \tilde{\rho}^{2}\right)}{\theta_{1}\left((-i / 2) \ln \zeta-\tilde{\beta} / 2-(i / 2) \ln \tilde{\rho}, \tilde{\rho}^{2}\right)}\right),
\end{aligned}
$$

where the second argument of the elliptic $\theta_{1}$ function is its nome, usually denoted by $q$. For efficient numerical evaluation of $\theta_{1}$, it is convenient to use the rapidly convergent representation

$$
\theta_{1}(s, q)=2 q^{1 / 4} \sum_{n=0}^{\infty}(-1)^{n} q^{n(n+1)} \sin (2 n+1) s .
$$

The function $f(\zeta, \tilde{\rho})$ is dependent on time $t$ only through $\tilde{\rho}$. When $\tilde{\rho}=\rho$, the steady state value $f$ is determined by the boundary conditions $\operatorname{Im} f=0$ on $|\zeta|=\rho$ and

$$
U \operatorname{Re} f=\mathscr{B} \kappa_{s},
$$

on $\zeta=e^{i v}$, where $\kappa_{s}$ is the steady state curvature and $\mathscr{B}=b^{2} T / 12 \mu V a^{2}$. Here $f$ is conveniently expressible as a Laurent series:

$$
f(\zeta, \rho)=a_{0}+\sum_{n=1}^{\infty}\left(a_{n} \zeta^{n}+a_{n} \rho^{2 n} \xi^{-n}\right),
$$

where the coefficients $a_{n}$ are all real. These have been deter- mined earlier. ${ }^{15}$ The complex velocity potential is decomposed into

$$
W=W_{0}(\zeta, \tilde{\rho}, \tilde{\beta})+\widetilde{W}_{1}(\zeta, t)+i[(U-1) / \pi](\pi-\tilde{\beta}),
$$

where

$$
W_{0}=-2 \frac{(U-1)}{\pi} \log \left(\frac{\theta_{4}((-i / 2) \ln \zeta, \tilde{\rho})}{\theta_{4}((-i / 2) \ln \xi-\tilde{\beta} / 2 \tilde{\rho})}\right)
$$

and the second argument of the elliptic $\theta_{4}$ function is its nome. For efficient numerical evaluation of this function, it is convenient to use the following rapidly convergent series representation:

$$
\theta_{4}(s, q)=1+2 \sum_{n=1}^{\infty}(-1)^{n} q^{n^{2}} \cos 2 n s .
$$

For the steady problem $\widetilde{W}_{1}$ and $\tilde{z}$ are each equal to zero. Further, for the steady state, $\tilde{\beta}=\pi$ and $\tilde{\rho}=\rho$, which is a characteristic of the size of the bubble. Inserting (57) and (62) into Eqs. (23) and (37), we find that

$$
\begin{aligned}
& \tilde{\phi}_{1}+U \tilde{x}_{1}=\mathscr{B} \kappa_{1}, \\
& \tilde{\psi}_{1_{v}}=y_{v} x_{1_{s}}-x_{v} y_{1_{r}},
\end{aligned}
$$

where

$$
\begin{aligned}
& \kappa_{1}=-\left(x_{v}^{2}+y_{v}^{2}\right)^{-3 / 2}\left\{y_{v v} x_{1_{v}}-x_{v v} y_{1_{v}}\right. \\
&+x_{v} y_{1_{v v}}-y_{v} x_{1_{v}} \\
&-3\left[\left(x_{v} x_{1_{v}}+y_{v} y_{1_{v v}}\right) /\left(x_{v}^{2}+y_{v}^{2}\right)\right] \\
&\left.\quad \times\left(x_{v} y_{v v}-y_{v} x_{v v}\right)\right\}, \\
& \tilde{\phi}_{1}+i \tilde{\psi}_{1}=\widetilde{W}_{1}, \\
& \tilde{x}_{1}+i \tilde{y}_{1}=\tilde{z}_{1}, \\
& x_{1}+i y_{1}=\tilde{z}_{1}+z_{0_{\tilde{\beta}}} \beta_{1}+\left(z_{0_{\tilde{p}}}+f_{\tilde{\rho}}\right) \rho_{1}, \\
& x+i y=z_{0}(\zeta, \rho, \pi)+f(\zeta, \rho),
\end{aligned}
$$

where $\beta_{1}$ and $\rho_{1}$ are the perturbation of $\tilde{\rho}$ and $\tilde{\beta}$ about the stationary values $\rho$ and $\pi$, respectively, and the subscripts $\tilde{\beta}$ and $\tilde{\rho}$ denote derivatives with respect to these two variables at the stationary values. Since $\operatorname{Im} \tilde{z}_{1}$ and $\operatorname{Im} \widetilde{W}_{1}$ are each zero on $|\zeta|=\tilde{\rho}$, each of these quantities are also zeroon $|\zeta|=\rho$ to leading order. Then it follows that for a general disturbance, $\tilde{z}_{1}$ and $\widetilde{W}_{1}$ each have Laurent series representations of the form

$$
b_{0}+\sum_{n=1}^{\infty}\left(b_{n} \zeta^{n}+b_{n}^{*} \rho^{2 n} \xi^{-n}\right),
$$

where $b_{0}$ is real. For symmetric disturbances, the $b_{n}$ are all real and for antisymmetric disturbances, $b_{0}$ is zero, while the other $b_{n}$ are purely imaginary. From the real and imaginary part of (72) on $\xi=e^{i v}$, we can express each of $\tilde{x}_{1}$ and $\tilde{\phi}_{1}$ in a cosine series for symmetric disturbances and sine series for the antisymmetric disturbances. For $\tilde{y}_{1}$ and $\tilde{\psi}_{1}$, the opposite is true. From the representation of $z_{0}$ in terms of $\rho$ and $\beta$, it is not difficult to deduce from (65) and (66) that $\beta_{1}=0$ for symmetric disturbances and $\rho_{1}=0$ for antisymmetric disturbances. Now we look for normal mode solutions to (65) and (66). For symmetric disturbances, we have 


$$
\begin{aligned}
& \tilde{x}_{1}=e^{\sigma t}\left(c_{0}+\sum_{n=1}^{\infty} c_{n}\left(1+\rho^{2 n}\right) \cos n v\right), \\
& \tilde{y}_{1}=e^{\sigma t}\left(\sum_{n=1}^{\infty} c_{n}\left(1-\rho^{2 n}\right) \sin n v\right), \\
& \tilde{\phi}_{1}=e^{\sigma t}\left(d_{0}+\sum_{n=1}^{\infty} d_{n}\left(1+\rho^{2 n}\right) \cos n v\right), \\
& \tilde{\psi}_{1}=e^{\sigma t}\left(\sum_{n=1}^{\infty} d_{n}\left(1-\rho^{2 n}\right) \sin n v\right) .
\end{aligned}
$$

For antisymmetric disturbances,

$$
\begin{aligned}
& \tilde{x}_{1}=e^{\sigma t}\left(\sum_{n=1}^{\infty} c_{n}\left(1+\rho^{2 n}\right) \sin n v\right), \\
& \tilde{y}_{1}=e^{\sigma t}\left(-\sum_{n=1}^{\infty} c_{n}\left(1-\rho^{2 n}\right) \cos n v\right), \\
& \tilde{\phi}_{1}=e^{\sigma t}\left(\sum_{n=1}^{\infty} d_{n}\left(1+\rho^{2 n}\right) \sin n v\right), \\
& \tilde{\psi}_{1}=e^{\sigma t}\left(-\sum_{n=1}^{\infty} d_{n}\left(1-\rho^{2 n}\right) \cos n v\right) .
\end{aligned}
$$

In either case, if we truncate the infinite series representation in (73)-(80) and satisfy Eqs. (65) and (66) at a discrete set of points on $\zeta=e^{i v}$, we end up with a matrix eigenvalue problem involving real matrices, which is solved as described in the next section.

We note that it is not necessary for any eigenvector comprising the components $c_{n}, d_{n}$, and $\rho_{1}$ or $\beta_{1}$ to be real even though each of the functions $\tilde{x}_{1}, \tilde{y}_{1}, \tilde{\phi}_{1}$, and $\tilde{\psi}_{1}$ defined by (68) and (69) and the constants $\rho_{1}$ and $\beta_{1}$ are real valued on $\zeta=e^{i v}$. This is because, for a general time-dependent problem, each of these functions as well as $\rho_{1}$ and $\beta_{1}$ are written as sums over all the eigenfunctions, each of which have the form (73)-(76), or (77)-(80). Although the sum has to be real, each eigenfunction can be complex.

\section{NUMERICAL PROCEDURE}

For symmetric modes, we truncate the expressions (73) and (74) to $N-1$ terms, i.e., we set $c_{j}=0$ for $j \geqslant(N-1)$. Equations (75) and (76) are truncated to $N$ terms so that $d_{j}$ $=0$ for $j \geqslant N$. The representation for the steady state function $f$ in (61) contained $N_{s} a_{n}$ terms, obtained from earlier work. ${ }^{15}$ Equations (65) and (66) are satisfied at $N+1$ points on the upper half semicircle $\zeta_{k}=e^{i k \pi / N}$, where $k$ is an integer ranging from 0 to $N$. Because of the assumed symmetry, it is not necessary to satisfy (65) and (66) at any points in the lower half semicircle. We thus obtain a matrix eigenvalue problem of dimension $2 N$, where the eigenvector $X$, written as a column, is given by

$$
X^{T}=\left(\rho_{1}, c_{0}, c_{1}, c_{2}, \ldots, c_{N-2}, d_{0}, d_{1}, \ldots, d_{N-1}\right) .
$$

The size of the system is reduced by half by noticing the block structure of the eigenvalue problem given by

$$
\left(\begin{array}{cc}
A_{1} & A_{2} \\
-\sigma B_{3} & A_{4}
\end{array}\right)\left(\begin{array}{l}
X_{1} \\
X_{2}
\end{array}\right)=0
$$

where $X_{1}^{T}=\left(\rho_{1}, c_{0}, \ldots, c_{N-2}\right)$ and $X_{2}^{T}=\left(d_{0}, \ldots, d_{N-1}\right)$. From (82), we can eliminate $X_{2}$ to get

$$
-B_{3}^{-1} A_{4} A_{2}^{-1} A_{1} X_{1}=\sigma X_{1} \text {. }
$$

This was solved by using a standard eigenvalue solver. Consistency of the eigenvalues and eigenvectors were checked by doubling $N$. The steady state truncation $N_{s}$ was also varied to check the results. Here $N=256$ and $N_{s}=128$ were found adequate for at least three figure accuracy in the eigenvalues for all the branches in the entire range of calculations reported except when $\mathscr{B} \leqslant 0.002$. For these smaller values of $\mathscr{B}$, $N=512$ and sometimes $N=1024$ were necessary to find at least a two figure accuracy in the eigenvalues up to $\mathscr{B}=0.0006$, beyond which the calculations were not reliable.

For antisymmetric modes, we truncate the summation in (77) and (78) to $N-1$ terms and in (79) and (80) to $N$ terms. Equations (65) and (66) are each satisfied at $N$ points on the unit semicircle $\zeta_{k}=e^{i v_{k}}$, where $\nu_{k}=\pi /$ $(2 N)+k \pi / N$ and $k$ ranges from 0 to $N-1$. The points $\zeta=1$ and $\zeta=-1$ are avoided because (65) and (66) are automatically satisfied at those points for antisymmetric disturbances. Because of the assumed antisymmetry of the disturbance, it is not necessary to satisfy (65) and (66) at $\xi$ points on the lower half semicircle. Once again, we have a generalized matrix eigenvalue problem of the form of (82), except $X_{1}^{T}=\left(\beta_{1}, c_{1}, \ldots, c_{N-1}\right)$ and $X_{2}^{T}=\left(d_{1}, \ldots, d_{N}\right)$ in this case. It was found that $N=512$ was adequate to give three figure accuracy for the three smallest eigenvalues over the range of calculations reported. The antisymmetric modes did not appear to be as sensitive to the truncation for small $\mathscr{B}$ as the symmetric disturbances were.

\section{NUMERICAL RESULTS}

All the eigenmodes were found to preserve the area of the bubble, which is expected since the flux of fluid at infinity is assumed to be the same. This provides a check on the code. The numerical calculations always gave two zero eigenvalues for the symmetric case, each with the same eigenmode which corresponds to a pure translation along the $x$ axis. The existence of a zero eigenvalue corresponding to a pure translation is caused by the invariance of the bubble shape to displacement in the $x$ direction. But the existence of a double eigenvalue needs some explanation, and the reason is as follows. For any steady state bubble, there is a neighboring steady bubble with slightly different area which moves with a slightly different speed. This second bubble, in the rest frame of the first, corresponds to a disturbance to the first bubble linear in time, and not of the form $e^{\sigma t}$. It is a well known result in spectral theory that the existence of such a solution to the disturbance equations requires that the stability operator for $\sigma=0$ has a generalized eigenvector, i.e., the zero eigenvalue must be at least a double root with only one independent eigenvector.

For the antisymmetric case, none of the numerically calculated eigenvalues were zero, which is consistent with our earlier findings ${ }^{15}$ that nonsymmetric steady bubbles do not exist as a continuation from the symmetric steady bubbles. However, as the bubbles became smaller, one of the eigenvalues approached zero, which is consistent with analytical results in Sec. II where it was shown that there is a zero eigenvalue for antisymmetric disturbances corresponding to the displacement of the bubble in the $y$ direction. When the wall 


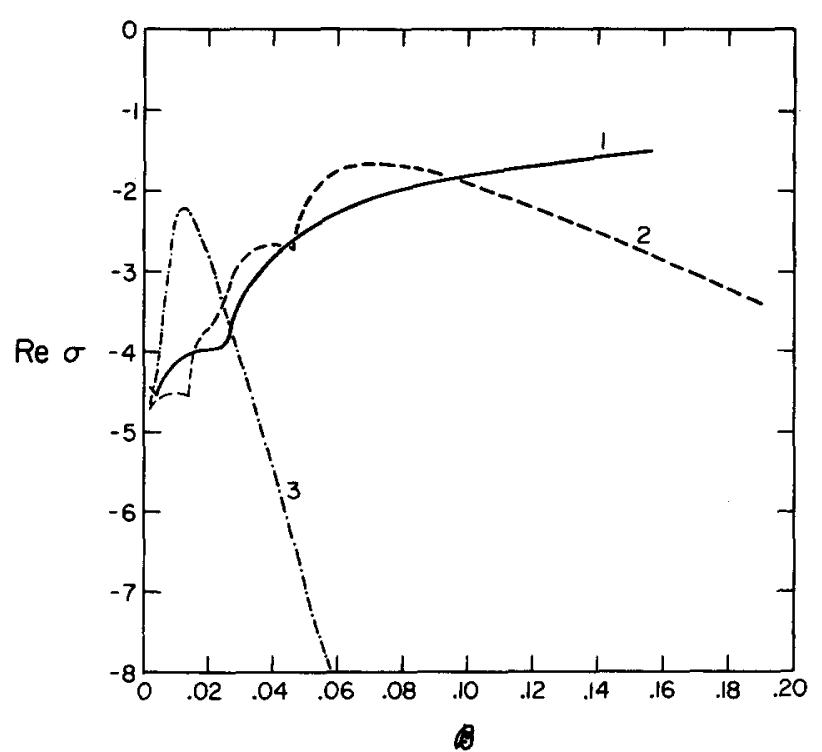

FIG. 4. Growth rate for the least stable symmetric disturbance for the McLean-Saffman bubbles. The curves marked 1, 2, and 3 correspond to area $J=3.8854,1.3854$, and 0.3854 , respectively. Note that the curves 1,2 , and 3 have discontinuous slopes at some values of $\mathscr{B}$ either because the least stable modes on two sides of the discontinuity are different or because two modes with real eigenvalue $\sigma$ coalesce to form a pair of complex conjugate $\sigma$.

effect is introduced, $y$ translation of the bubble becomes an eigenmode corresponding to a nonzero eigenvalue.

Now we examine the stability features of the main branch $^{14}$ (McLean-Saffman branch) of bubble solutions. Figure 4 shows the real part of the eigenvalue, i.e., the growth rate, for the least stable symmetric eigenmode for three different values of area $J$ for $\mathscr{B}$ ranging from 0.002 to 0.200 . The curves have discontinuous slopes at some points either because two real eigenvalues coalesced to give a complex conjugate pair of eigenvalues or because the least stable mode on the left and right of any discontinuity point are different. Also, in Fig. 4, for $J=1.3854$ and 0.3854 , the least

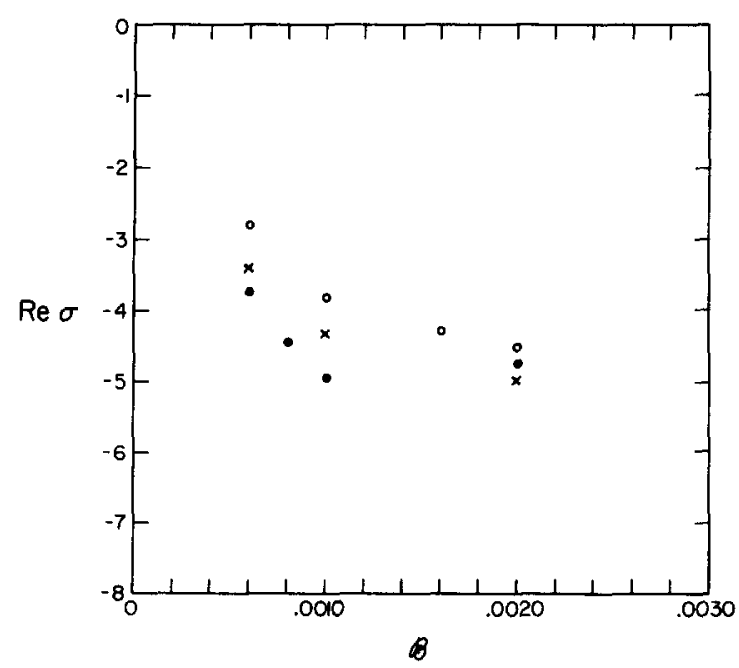

FIG. 5. Growth rate for the least stable symmetric mode for the McLeanSaffman bubbles for small isolated values of $\mathscr{B}$ for relatively large values of $J$. The open circles, crosses, and closed circles correspond to $J=3.8854$, 2.7654 , and 1.3854 , respectively.

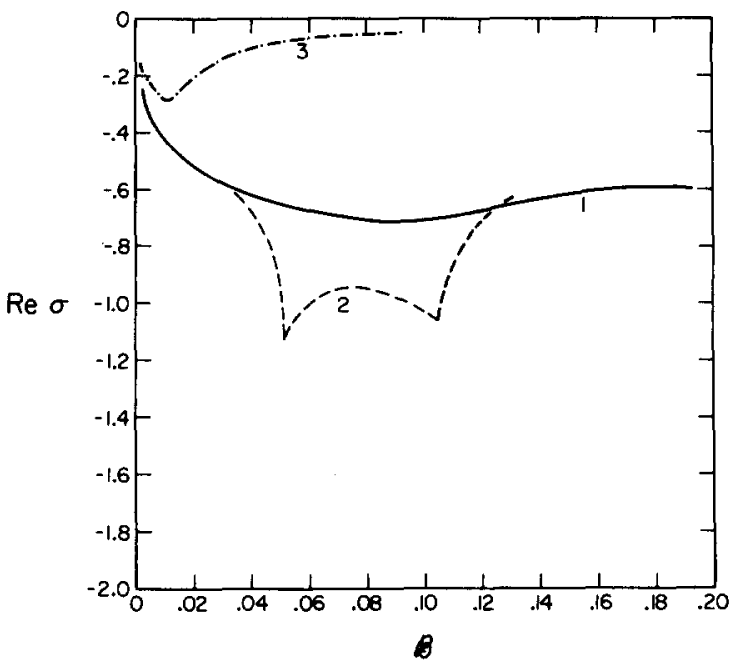

FIG. 6. Growth rate for the least stable antisymmetric disturbance for the McLean-Saffman bubbles. The curves marked 1, 2, and 3 correspond to area $J=3.8854,1.3854$, and 0.3854 , respectively. Note that the curves 1 and 2 coincide for small values of $\mathscr{B}$. The curve 2 has discontinuous slope because of two real $\sigma$ coalescing to form one pair of complex conjugate $\sigma$.

stable eigenvalues appear to depend on surface tension linearly for large values of surface tension where the bubble shapes are more or less circular. The eigenvalues in this range of $\mathscr{B}$ were found to be real. If the effect of the walls were totally ignored, Eq. (16) implies that the largest eigenvalue, excluding $\sigma=0$, would obey the relation $\sigma_{2}=-139.6 \mathscr{B}$ when $J=0.3854$. Measurement of the slope of the corresponding curve in the linear regime in Fig. 4 shows $\sigma_{2}=-137 \mathscr{B}$, which is close to that predicted by (16). Similar agreements were noted for $\sigma_{3}$ and $\sigma_{4}$. For smaller values of area $J$, better agreement with Eq. (16) was noted. Figure 5 shows the real part of the eigenvalue for the least stable symmetric eigenmode for small values of $\mathscr{B}$. From extrapolation, it appears that the bubble remains stable in the limit of zero surface tension. However, we note a significant dependence on the area of the bubble for relative-

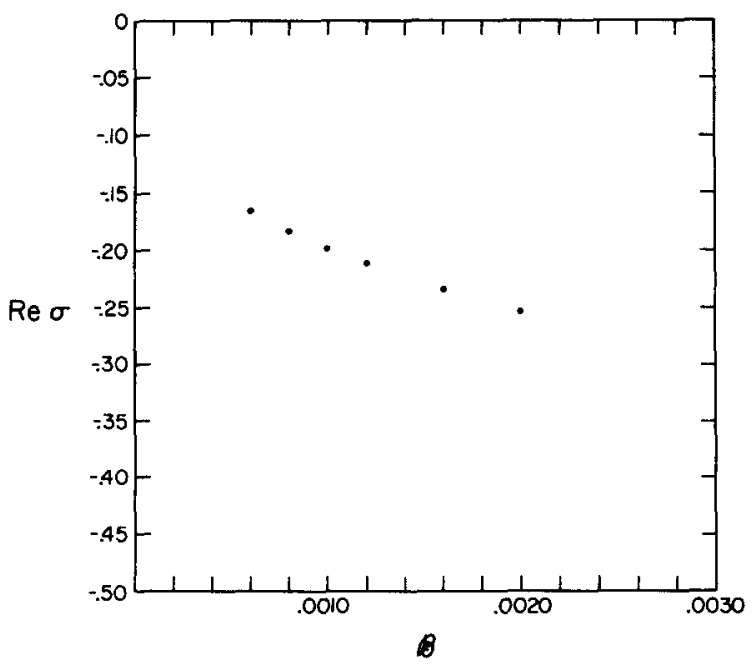

FIG. 7. Growth rate for the least stable antisymmetric mode (the vertical translation mode) for the McLean-Saffman bubble for relatively large values of $J$. The plots for $J=3.8854,2.7654$, and 1.3854 are indistinguishable and are all shown here. 


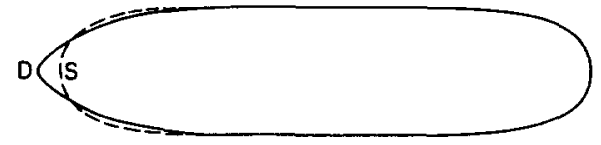

FIG. 8. The effect of the least stable symmetric mode on the steady state McLean-Saffman bubble with $J=3.8854$ and $\mathscr{B}=\mathbf{0 . 0 0 2}$. The letters $S$ and $\mathrm{D}$ on the figure denote steady and disturbed configurations. A similar effect was observed for other values of $J$ and $\mathscr{B}$.

ly large bubbles. Thus it is not possible to extrapolate these results to infinite bubbles that would correspond to the McLean-Saffman finger. Figure 6 shows the least stable antisymmetric mode for different values of $J$ and $\mathscr{B}$. The curves marked 1 and 2 are indistinguishable for small values of $\mathscr{B}$ suggesting that for large enough $J$, the decay rate of this mode is independent of $J$ for small $\mathscr{B}$. From (16), $\sigma_{1}=0$ is a possible eigenvalue for antisymmetric disturbance that corresponds to a translation in the $y$ direction, which is possible when the wall effects are ignored. When the wall effects are considered, this is no longer a neutral eigenvector; however, this translation is the least stable antisymmetric disturbance for a wide range of area and surface tension. With decreasing bubble size the decay rate for this disturbance approaches zero as can be seen in Fig. 6 . Thus the cell wall effects become increasing unimportant for decreasing bubble size as expected. Figure 7 shows the growth rate for the least stable antisymmetric mode for $J=3.8854$. Almost identical dependence was noted for $J=2.7654$ and $J=1.3854$ suggesting that there is, for the McLean-Saffman finger, a similar mode which is stable. For $\mathscr{B} \leqslant 0.0006$, for $N \leqslant 512$ and $N_{s}=258$ we could not obtain two digit accuracy in the least stable eigenmode as comparison with the results obtained by doubling $N$ showed. Figure 8 shows the effect of the least stable symmetric mode on the steady state bubble for $J=3.8854$ and $\mathscr{B}=0.002$. Other symmetric modes, which are more stable, are also found not to disturb the front end of the finger to any appreciable extent. In the limit of infinite bubble size, the front half of the bubble tends to a finger. However, since the least stable modes are found to affect the back of the bubble, there are no corresponding disturbances on the finger since the finger does not have a rear end. This explains the appreciable dependence of eigenvalue on area in Fig. 5.

Now we consider the first Romero-Vanden-Broeck branch. For most values of $\mathscr{B}$, two unstable modes, one sym-

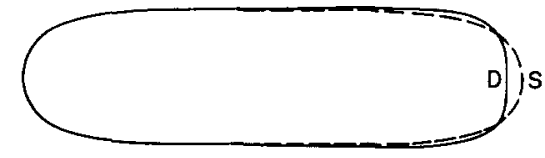

FIG. 9. The effect of the unstable symmetric mode (tip breaking) on the first Romero-Vanden-Broeck bubble with $J=3.8854$ and $\mathscr{B}=0.004$. The letters $S$ and $D$ denote steady and disturbed configurations, respectively.

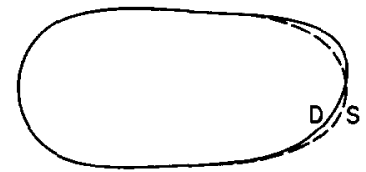

FIG. 10. The effect of the unstable antisymmetric mode on the first Romero-Vanden-Broeck bubble with $J=2.7654$ and $\mathscr{B}=0.0005$. The letters $\mathrm{S}$ and $\mathrm{D}$ in the figure denote steady and disturbed configurations, respectively.

metric and one antisymmetric, were found. One of them is a symmetric tip-breaking mode. Figure 9 shows the effect of that mode on a bubble with area $J=3.8854$ and $\mathscr{B}=0.004$. The other unstable mode was antisymmetric and the effect of this on a steady bubble of area $J=2.7654$ with $\mathscr{B}=0.0005$ is shown in Fig. 10. The growth rate of the unstable symmetric mode is shown if Fig. 11 for $J=3.8854,2.7654,1.3854$, and 0.3854 . This eigenvalue was always found to be real. The growth rate of the unstable antisymmetric mode is plotted in Fig. 12 for different values of $J$. In this case, the eigenvalue $\sigma$ becomes complex when $\mathscr{B}$ exceeds some critical value depending on $J$ where the curves in Fig. 12 have a discontinuity in slope. In some small neighborhood of the critical points, one eigenmode besides the unstable mode whose growth rate is shown in Fig. 12 was found to change stability characteristics from being stable to unstable before coalescing with the other mode at the critical point. For values of $\mathscr{B}$ larger than the right end point of each of the curves, no steady states could be found. In earlier work, ${ }^{15}$ we reported steady state bubble characteristics on the first Romero-Vanden-Broeck branch only for $J=3.8854$. This branch of solutions has been subsequently continued to smaller areas. For small area, the bubble is found to be elongated in a direction perpendicular to the channel flow. Further, with increasing surface tension, the bubble curvature becomes negative and the results presented in Fig. 11 show that these small bubbles are

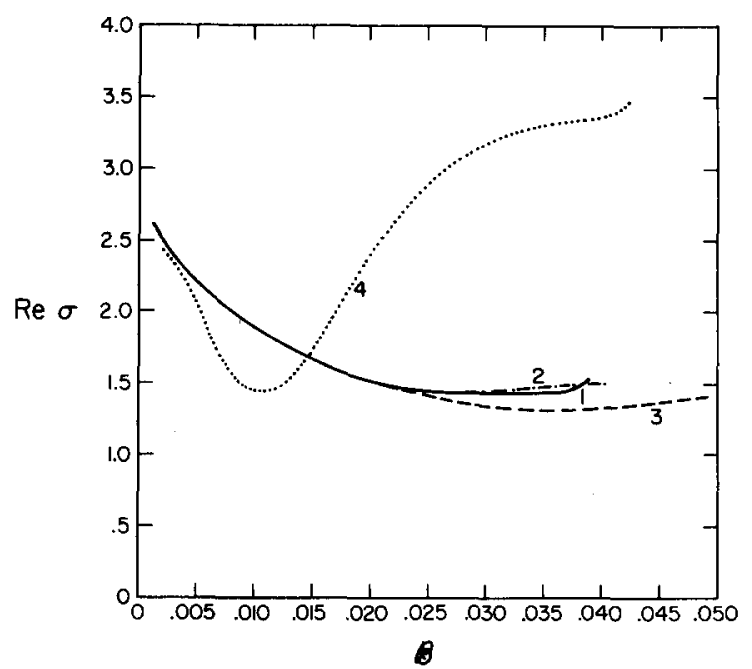

FIG. 11. The growth rate of the unstable symmetric mode (tip breaking) on the first Romero-Vanden-Broeck branch of bubbles for different $J$. The curves marked $1,2,3$, and 4 correspond to $J=3.8854,2.7654,1.3854$, and 0.3854 , respectively. 


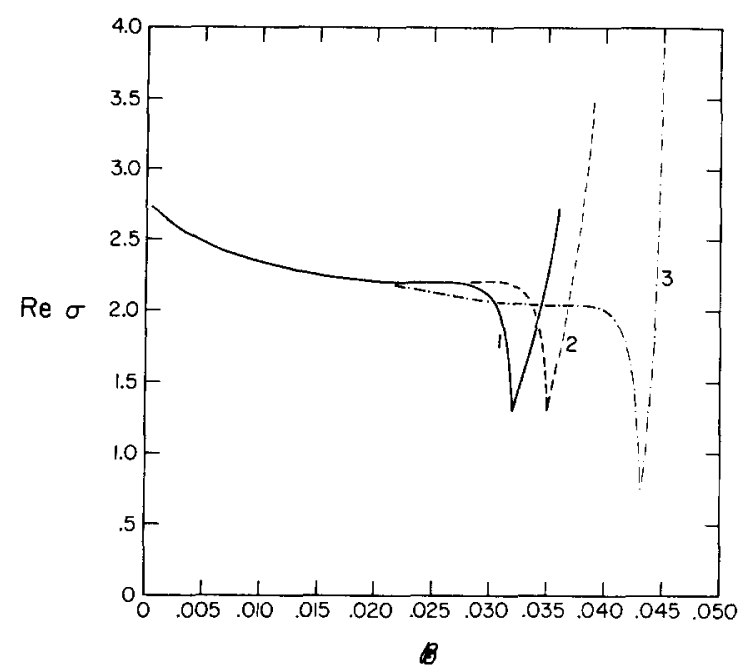

FIG. 12. The growth rate for the most unstable antisymmetric mode on the first Romero-Vanden-Broeck branch of bubbles for different $J$. The curves marked 1,2 , and 3 correspond to $J=3.8854,2.7654$, and 1.3854 , respectively. Each of the curves has discontinuous slope at some relatively large $\mathscr{B}$, where two modes with real eigenvalue coalesce to give a pair of conjugate eigenvalues.

very unstable. Since the unstable modes affect only the front half of the bubble, it is clear that similar modes of instability arise for the finger. Figures 13 and 14 show the unstable eigenvalues as functions of $\mathscr{B}^{2 / 3}$ for $J=3.8854$ for symmetric and antisymmetric branches and in each case the curve appears to be linear and passes through 2.8 as predicted by the analytic theory ${ }^{12}$ for the finger. This is also in rough agreement with the numerical results of Kessler and Levine ${ }^{19}$ for the finger. Further, the curves are found to be right on top of each other when $J=2.7654$ suggesting that the finger limit has been approached when $J=3.8854$. From the slope of the linear curves in Fig. 13, we conclude that, for small $\mathscr{B}$,

$$
\sigma=2.8-19 \mathscr{B}^{3 / 2}
$$

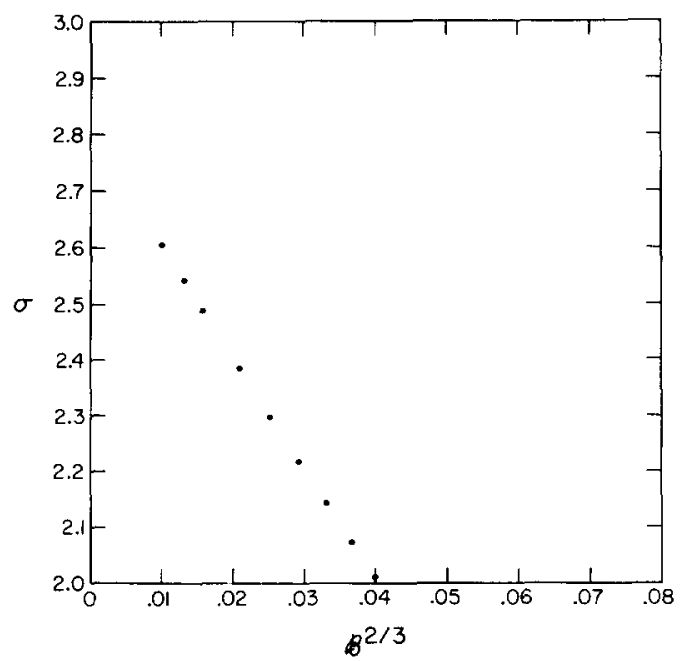

FIG. 13. Dependence of $\sigma$ on $\mathscr{B}^{3 / 2}$ for small $\mathscr{B}$ for the unstable symmetric mode for the first Romero-Vanden-Broeck branch of bubbles. Here, $J=3.8854$ but the $J=2.7654$ plot is indistinguishable from this.

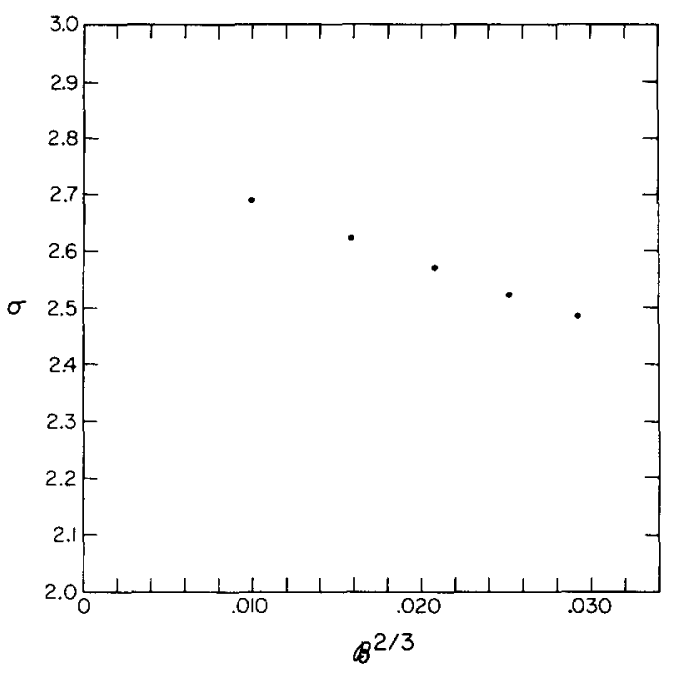

FIG. 14. Dependence of $\sigma$ on $\mathscr{B}^{3 / 2}$ for small $\mathscr{B}$ for the unstable antisymmetric mode for the first Romero-Vanden-Broeck branch of bubbles. The plots for $J=3.8854$ and 2.7654 are indistinguishable.

for the symmetric tip-breaking unstable mode, and, from Fig. 14,

$$
\sigma=2.8-11 \mathscr{B}^{3 / 2}
$$

for the unstable antisymmetric mode.

In the original steady state calculations, ${ }^{15}$ we reported finding only a branch of bubble solutions that in the limit of large area corresponds to the first Romero-Vanden-Broeck branch of finger solutions. Subsequent work showed that there was another branch of bubble solutions that in the limit of large area corresponds to the second Romero-VandenBroeck branch of finger solutions. Here we present stability results on this branch for a limited range of parameters. From our limited calculations two unstable modes were detected for each of the symmetric and antisymmetric modes. Figure 15 shows the dependence of the real part of the two

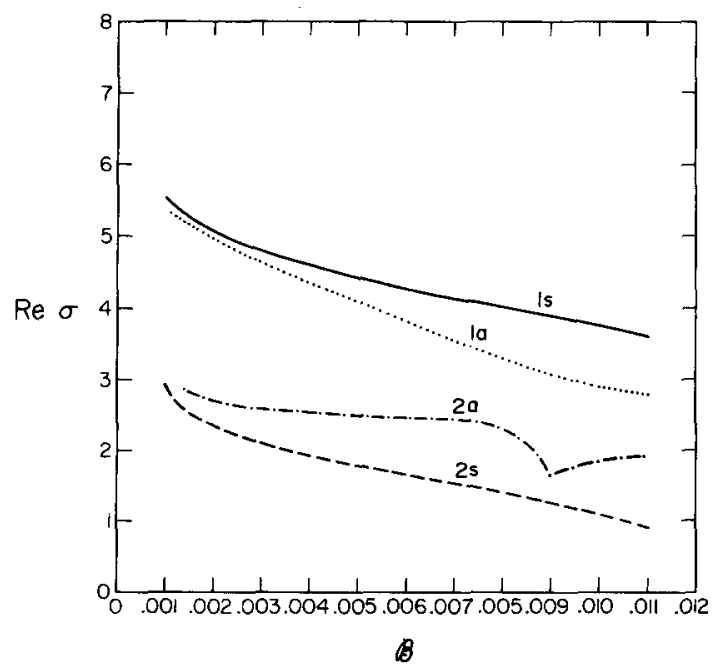

FIG. 15. Dependence of the growth rate on $\mathscr{B}$ for the unstable symmetric and antisymmetric modes for $J=3.8854$ for the second Romero-VandenBroeck bubble solution. The numbers 1 and 2 distinguish the two unstable modes and the letters $a$ and $s$ denote symmetric and antisymmetric modes. 
unstable symmetric and antisymmetric eigenvalues on surface tension for $J=3.8854$. Each of these modes were found to affect only the front half of the bubble, implying that these instability modes exist for fingers as well. This is also consistent with the numerical findings of Kessler and Levine ${ }^{19}$ as well as the analytical work of Tanveer ${ }^{12}$ on the stability of fingers.

\section{DISCUSSIONS AND CONCLUSIONS}

We have presented details for the linear stability of bubbles in a Hele-Shaw cell. Except for very small surface tension, where our numerical procedure breaks down, we find that the bubbles on the McLean-Saffman branch of solutions are stable up to some moderate large bubble size, whereas the Romero-Vanden-Broeck branch of bubble solutions are unstable. Comparison of the unstable eigenvalues of the first Romero-Vanden-Broeck branch of bubble solutions for large bubble area with that of the limiting value for the finger worked out analytically ${ }^{12}$ shows good agreement. Further for small eigenvalues, we are able to calculate the spectrum exactly and show that the spectrum has entirely different characteristics with and without surface tension. The effect of transcendentally small terms in surface tension is inferred. Further, for bubbles of arbitrary size, we find that for zero surface tension, the spectrum is the entire complex plane and therefore the bubbles are unstable. A regular perturbation scheme appears to suggest that the bubbles remain unstable. It then appears that the only way our numerical results of a discrete stable spectrum can be reconciled with the zero surface tension case is for transcendentally small terms in surface tension to have the effect of perturbing the zero surface tension modes such that the bubble boundary no longer remains analytic except when the eigenvalues belong to a discrete set of nonpositive values. An appropriate theory to show that this is the case can be constructed similar to the earlier ${ }^{12}$ theory for fingers. This is left for the future.

\section{ACKNOWLEDGMENT}

This work was supported by the Department of Energy, Office of Basic Energy Sciences Grant No. DE AT03-76ER72012.

${ }^{1}$ F. F. Craig, The Resenvoir Engineering Aspects of Water Flooding (Society of Petroleum Engineers, New York, 1971).

${ }^{2}$ L. H. Ungar and R. A. Brown, Phys. Rev. B 29, 1367 (1984).

${ }^{3}$ B. B. Mandelbrot, The Fractal Geometry of Nature (Freeman, San Francisco, 1982).

${ }^{4}$ J. W. McLean and P. G. Saffman, J. Fluid Mech. 102, 455 (1980).

${ }^{5}$ L. Romero, Ph.D. dissertation, California Institute of Technology, 1982. 'J.-M. Vanden-Broeck, Phys. Fluids 26, 2033 (1983).

${ }^{7}$ D. Kessler and H. Levine, Phys. Rev. A 32, 1930 (1985).

${ }^{8}$ R. Combescot, T. Dombre, V. Hakim, Y. Pomeau, and A. Pumir, Phys. Rev. Lett. 56, 2036 (1986).

${ }^{9}$ D. C. Hong and J. S. Langer, Phys. Rev. Lett. 56, 2032 (1986).

${ }^{10}$ B. Shraiman, Phys. Rev. Lett. 56, 2028 (1986).

'D. Bensimon, L. Kadanoff, B. Shraiman, and C. Tang, Rev. Mod. Phys. 58, 977 (1986).

${ }^{12}$ S. Tanveer, Phys. Fluids 30, 2318 ( 1987).

${ }^{13}$ P. G. Saffman and G. I. Taylor, Proc. R. Soc. London Ser. A 245, 312 (1958).

${ }^{14} \mathrm{~S}$. Tanveer, Phys. Fluids 29, 11 (1986)

${ }^{15}$ S. Tanveer, Phys. Fluids 30, 3 (1987).

${ }^{16}$ G. I. Taylor and P. G. Saffman, Q. J. Mech. Appl. Math. 12, 265 (1959).

${ }^{17} \mathbf{L}$. Kadanoff (private communication).

${ }^{18}$ S. Tanveer, Phys. Fluids 30, 1586 (1987).

${ }^{19}$ D. Kessler and H. Levine, Phys. Rev. A 33, 2632 (1986). 